\section{Management of band keratopathy with excimer phototherapeutic keratectomy: visual, refractive, and symptomatic outcome}

OG Stewart and AJ Morrell

pathology is common and may limit the extent of visual improvement postoperatively. Factors associated with the myopic shift seen in this series may be epithelial remodelling, concurrent ocular pathology, or inaccuracy of preoperative refractive data.

Eye (2003) 17, 233-237. doi:10.1038/

sj.eye. 6700327

Keywords: phototherapeutic keratectomy (PTK); excimer laser; band keratopathy; corneal degeneration

Introduction

Excimer Phototherapeutic keratectomy (PTK) has been found to be an effective treatment for a variety of superficial corneal disorders. Corneal surface irregularity, epithelial instability, and superficial opacification may all benefit from the procedure. $^{1-8}$

Band keratopathy is a relatively common superficial corneal degeneration in which deposition of extracellular granular material throughout the epithelial basement membrane, Bowman's layer, and anterior stroma may result in any combination of these problems.

Histological analysis shows that cases may be due to deposition of calcific, noncalcific, or mixed-type materials. ${ }^{9}$ Noncalcific material deposition may account for the poor response to chelating agents such as EDTA seen in some eyes. ${ }^{10}$

The indiscriminate ablative action of PTK means that treatment of all bands, independent of biochemical make-up, is possible. Excimer ablation allows precise tissue removal over
Department of

Ophthalmology

St James's University

Hospital

Leeds, West Yorkshire, UK

Correspondence:

OG Stewart

Department of

Ophthalmology

Leeds General Infirmary

Leeds LS2 9NS

UK

Tel: +44 1132432799

Fax: +44 1132926239

E-mail: owenstewart@

fsmail.net

Received: 1 October 2001 Accepted in revised form: 11 June 2002

This work was presented as a poster at the Royal College of Ophthalmologists Annual Congress Meeting in Birmingham, May 2001 
wide areas of cornea without trauma to adjacent tissue, resulting in smooth ablation zones. This is in contrast to manual superficial keratectomy where surface irregularities may occur. In addition, the depth and area of ablation is easily controlled, and thus PTK is an ideal modality of treatment for this condition.

Therapeutic excimer treatment tends to be restricted to large teaching units within the UK, and has been available in the Yorkshire region since late 1995. This study was to determine the visual and refractive outcome of patients undergoing PTK for band keratopathy since this time.

\section{Materials and methods}

Analysis was performed on 45 consecutive PTK procedures on 33 patients with band keratopathy. Patients were assessed as to whether the indication for PTK was for visual rehabilitation (visual group) or for ocular surface improvement to alleviate symptoms of pain or discomfort (nonvisual group). Further data including pre- and postoperative best-corrected visual acuity (BCVA), pre- and postoperative spherical equivalent (SE), and associated ocular or systemic pathology were collected. Pre- and postoperative spherical equivalents were subject to statistical analysis using the paired $t$-test.

All of the patients were recruited from the cornea and external eye disease clinic at St James's University Hospital, Leeds. All were treated by a single surgeon (AJM) during the period between January 1996 and December 1999. There were 23 males and 10 females. The age range of the patients was between 14 and 90 years (mean 68 years). A total of 21 patients had unilateral treatment, while 12 had bilateral treatment. The aim for PTK was visual rehabilitation in 33 eyes of 22 patients, and relief of symptoms in 12 eyes of 11 patients.

Treatment was performed with a Technolas-116 $193 \mathrm{~nm}$ excimer laser (Bausch \& Lomb). Mechanical epithelial debridement was followed by a central $5 \mathrm{~mm}$ ablation zone, centred on the visual axis. Blending of the ablative zone was performed out to $7 \mathrm{~mm}$ by multiple, overlapping satellite ablative zones. Calcium plaques due to rough bands were removed mechanically using a surgical blade, and methylcellulose was used as a masking agent for irregular surfaces. Adequate ablative depth was assessed by regular visualisation of the cornea between laser bursts down the operating microscope or by peri-operative slit-lamp examination. All patients were treated on an outpatient basis.

Post-operative treatment consisted of a therapeutic contact lens with topical antibiotics and NSAIDs. The contact lens was usually removed at $48 \mathrm{~h}$, and antibiotics were continued until full epithelial healing had occurred.

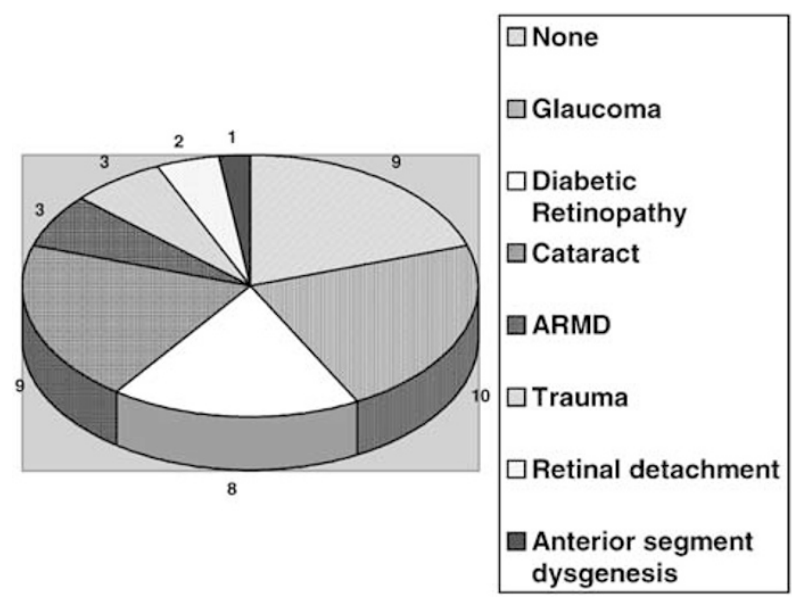

Figure 1 Associated ocular pathology in eyes with band keratopathy.

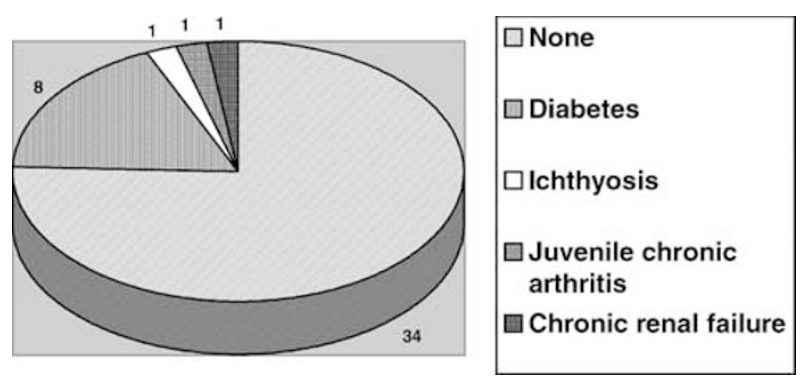

Figure 2 Associated systemic pathology in eyes with band keratopathy.

Follow-up in the clinic was at 48 h, 1, 4, and 12 weeks, and thereafter at 6-month intervals. The follow-up ranged from 13 months to 46 months (mean 24.3). Refraction was initially performed at 4 weeks, and then at each clinic visit. For the purposes of this study, the postoperative refraction was deemed to be at 12 months following PTK.

Associated ocular pathology was commonly seen in this series of patients. Only nine eyes (20\%) were deemed to be free of any other ocular condition (Figure 1). In contrast, related systemic conditions were infrequently seen (Figure 2).

\section{Results}

A total of 45 eyes of 33 patients underwent PTK for band keratopathy (Figure 3). In the visual group, there were 33 eyes of 22 patients, and in the nonvisual group 12 eyes of 11 patients. BCVA was improved or unchanged in 18/33 eyes $(55 \%)$ of the visual group, while there was loss of one or more lines of acuity in 15/33 (45\%) (Figure 4). 


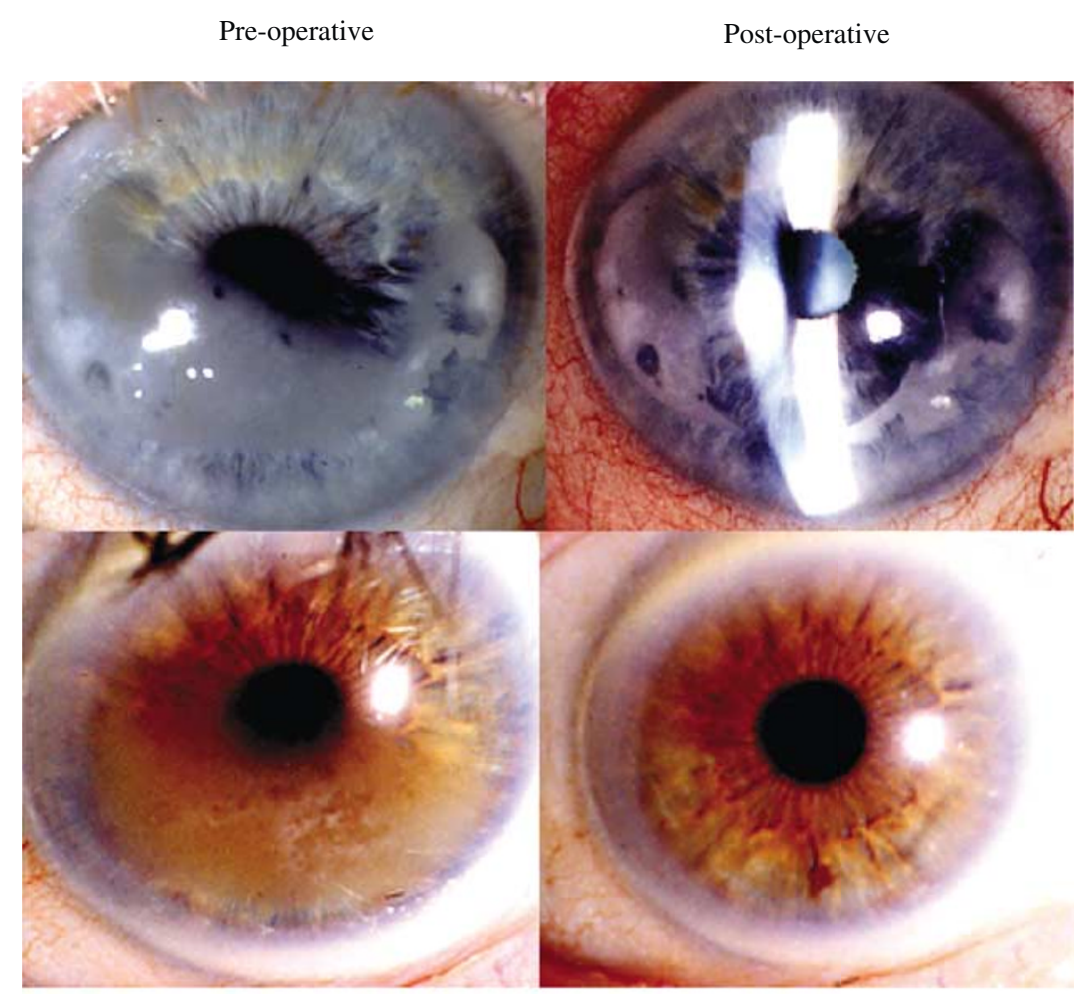

Figure 3 Band keratopathy: pre- and post-PTK appearances.

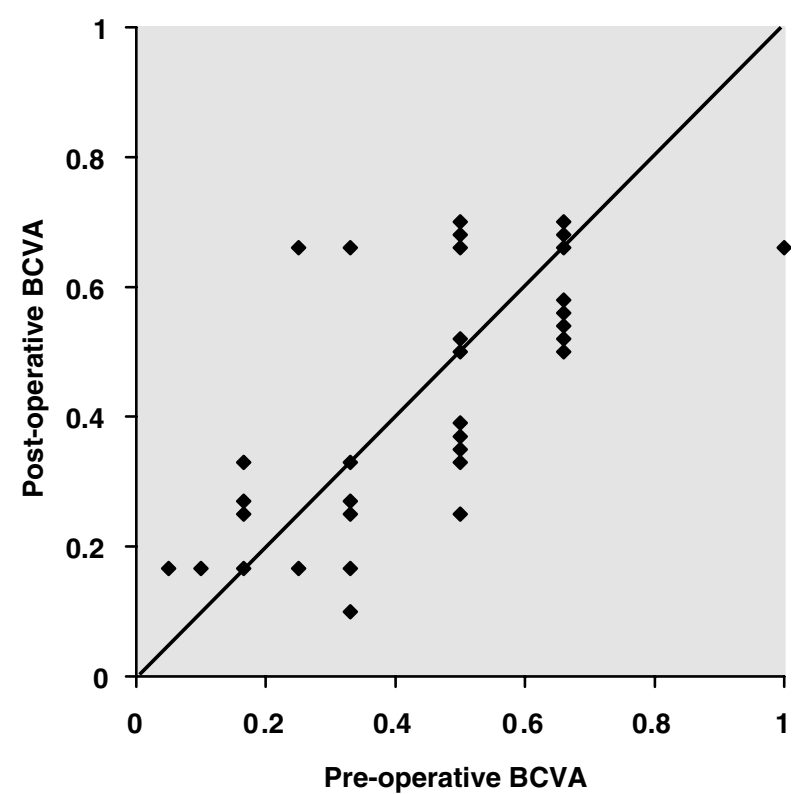

Figure 4 Pre- and postoperative BCVA 12 months following PTK (visual group).

Loss of BCVA was attributable to other ocular factors in 12/15 eyes (80\%) (Figure 5). The remaining eyes lost acuity due to subepithelial fibrosis and corneal opacification. In several cases, treatment was performed
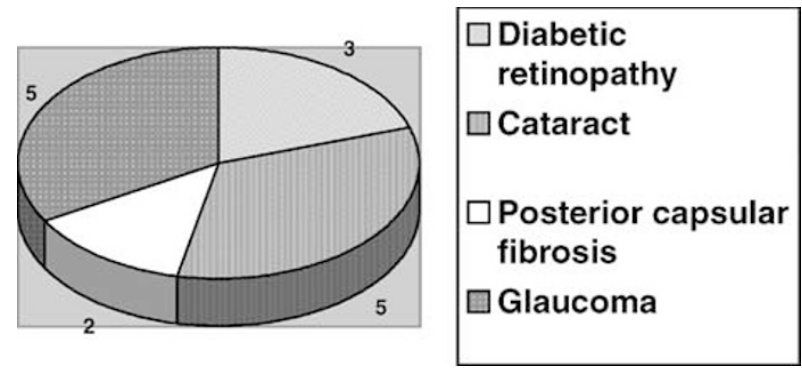

Figure 5 Other factors contributing to loss of BCVA.

prior to phacoemulsification or pan-retinal photocoagulation.

At 12 months, there was no significant refractive change $( \pm 1.00 \mathrm{D})$ in $16 / 33$ eyes $(48 \%)$. Out of 33 eyes $(45 \%), 15$ had a significant myopic shift (mean $-2.10 \mathrm{D})$, while 2/33 (6\%) had a significant hyperopic shift (mean $+1.12 \mathrm{D})$. The mean preoperative SE of patients within the visual group was +1.42 D (SD \pm 3.21$)$, and the mean 12-month postoperative SE was +0.32 D (SD $\pm 3.26)$. This revealed a significant myopic shift postoperatively $(P<0.5)$ (Figure 6). There were no significant complications in this group, and no cases of delayed epithelial healing.

In the nonvisual group, 10/12 (83\%) had significant improvement of symptoms of ocular pain and discomfort 


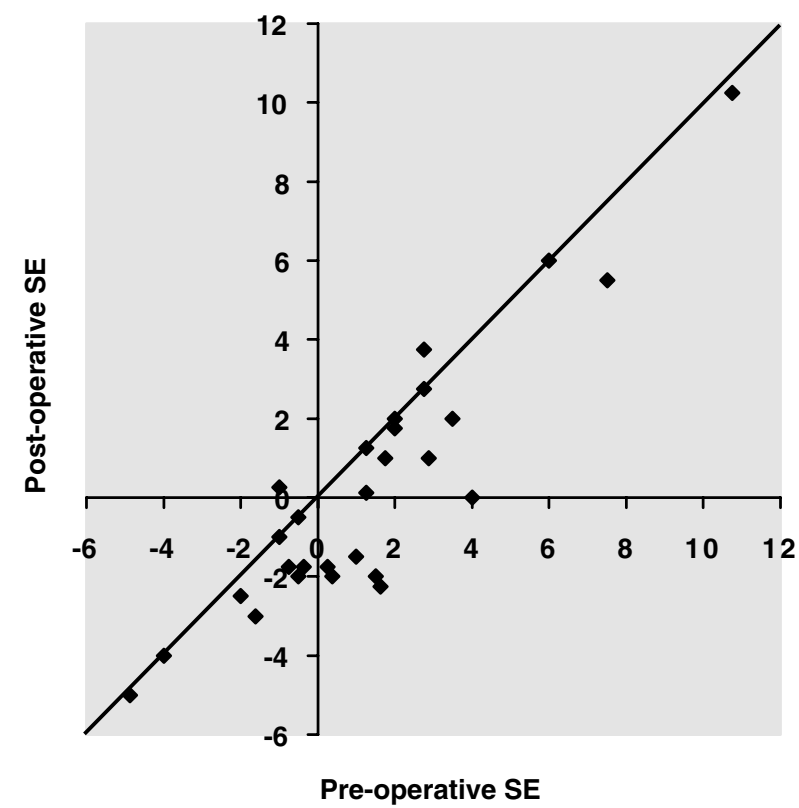

Figure 6 Pre- and postoperative spherical equivalent (visual group).

owing to surface irregularity, following PTK. All these eyes had visual acuities of hand movements or less, and all had rough bands. Mechanical debridement was performed in eyes with severe surface calcification prior to PTK. One eye developed bacterial keratitis, with a stromal abscess and hypopyon during epithelial healing. Re-epithelialisation tended to be slower in this group, with four eyes experiencing delayed epithelial healing more than 7 days post-treatment. This group, on the whole, required more prolonged therapeutic contact lens use, increased lubrication, and careful follow-up to ensure full epithelial cover.

No cases, deemed to have had successful treatment, had significant visual or symptomatic recurrence of band keratopathy during the period of follow-up.

\section{Discussion}

Band keratopathy may cause reduction of visual acuity and glare due to corneal opacification. It may also cause symptoms of pain and discomfort if epithelial breakdown occurs, which is typically seen in rough bands. In other studies, ${ }^{10,11}$ the effectiveness of PTK for both smooth and rough bands has been demonstrated. An improvement in both visual acuity and glare is expected in the vast majority of patients with smooth bands and, similarly, improved comfort is the common outcome of treatment in eyes with discomfort owing to rough bands. The visual results in this series seem less impressive at first glance, with $55 \%$ of eyes with improved or unchanged postoperative acuities at the 12month follow-up. However, concurrent ocular pathology was common in this group of eyes, and it was difficult to determine the extent of visual loss attributable to these other factors. Previous studies ${ }^{4,12}$ have shown PTK to be less effective in regard to visual outcome for band keratopathy than for some other superficial corneal disorders, mainly because of coexisting ocular disease.

Refractive changes following PTK can be variable, with significant myopic or hyperopic shifts postoperatively. ${ }^{4,13}$ Hyperopic shifts may be expected with deeper central ablations, and have been reported to occur in $40-81 \%$ of eyes following PTK for various corneal pathologies. ${ }^{1-4}$ $\mathrm{O}^{\prime}$ Brart $e t a l^{10}$ reported a mean hyperopic shift of $1.4 \mathrm{D}$ at 6 months following PTK in 32 eyes treated for visually significant band keratopathy. However, a number of patients may develop a myopic shift postoperatively, the reasons for which are unclear. Peripheral ablation may have a role in induced corneal steepening. Other possible factors include inaccuracy of preoperative refraction owing to corneal opacification, concurrent ocular pathology, for example, increasing nuclear sclerosis, or epithelial remodelling.

Ocular surface instability as a result of band keratopathy is effectively treated with PTK. The vast majority of patients with painful symptoms owing to rough bands can expect to have dramatic improvement in their symptoms following treatment. The accepted method of mechanical debridement of large calcific plaques prior to PTK allows for a shorter procedure time and for easier ablation. A masking agent is often necessary to achieve smooth ablation profiles. Deeper ablation is possible in blind eyes to achieve the desired effect without consideration of induced refractive error. Postoperative epithelial healing is often compromised in such eyes and careful supervision in the postoperative period is mandatory. One case in our series developed severe microbial keratitis despite the use of prophylactic topical antibiotics. Persistent epithelial defects may develop, necessitating prolonged therapeutic contact lens wear, preservative-free medication, or further surgical intervention in the form of botulinum toxin, tarsorrhaphy, or amniotic membrane transplantation.

Favourable ocular surface changes occur after PTK including increased tear film stability owing to attainment of a regular corneal surface, and probably through better mucin production by a healthier epithelium..$^{14}$ It is possible that improvements in these parameters may reduce the likelihood of band recurrence.

Significant recurrence of band keratopathy was not seen in this series with a mean follow-up of approximately 2 years following PTK. Recurrence is uncommonly seen, although eyes with intraocular silicone oil may be more at risk. ${ }^{10}$ 
Treatment may also be indicated prior to intraocular surgery. Improved visualisation during phacoemulsification or extracapsular cataract extraction ${ }^{15}$ may be achieved by initial PTK. Likewise, treatment in patients with suspected posterior segment disease allows easier examination, treatment, or surgery.

Access to therapeutic excimer treatment in the UK tends to be limited to teaching units, and an optional treatment is manual superficial keratectomy with EDTA chelation. While the majority of bands will respond, deposition of noncalcific material may limit the effectiveness of such treatment, ${ }^{10}$ and manual keratectomy may cause postoperative surface irregularities. However, the merit of PTK compared to EDTA chelation for band keratopathy requires a prospective controlled trial before conclusions regarding the relative outcome of these treatment modalities can be made.

In summary, excimer PTK is an effective procedure in the management of band keratopathy, whether it is performed for visual rehabilitation or for ocular surface improvement. However, concurrent ocular pathology is common, with limitation of visual improvement postoperatively, and patients need to be counselled accordingly.

\section{References}

1 Sher NA, Bowers RA, Zabel RW et al. Clinical use of the 193-nm excimer laser in the treatment of corneal scars. Arch Ophthalmol 1991; 109: 491-498.

2 Stark WJ, Chamon W, Kamp MT et al. Clinical follow-up of 193-nm ArF excimer laser photokeratectomy. Ophthalmology 1992; 99: 805-812.

3 Campos M, Nielsen S, Szerenyi K et al. Clinical follow-up of phototherapeutic keratectomy for treatment of corneal opacities. Am J Ophthalmol 1993; 115: 433-440.
4 Maloney RK, Thompson V, Ghiselli G et al. A prospective multicenter trial of excimer laser phototherapeutic keratectomy for corneal vision loss. The Summit PTK Study Group. Am J Ophthalmol 1996; 122: 149-160.

5 Bernauer W, De Cock R, Dart JK. Phototherapeutic keratectomy in recurrent corneal erosions refractory to other forms of treatment. Eye 1996; 10: 561-564.

6 Cavanaugh TB, Lind RM, Cutarelli PE et al. Phototherapeutic keratectomy for recurrent erosion syndrome in anterior basement membrane dystrophy. Ophthalmology 1999; 106: 971-976.

7 Gartry D, Kerr-Muir M, Marshall J. Excimer laser treatment of corneal surface pathology: a laboratory and clinical study. Br J Ophthalmol 1991; 75: 258-269.

8 Fagerholm P, Fitzsimmons TD, Örndahl MJ et al. Phototherapeutic keratectomy with the excimer laser: long term follow-up results in 166 treated eyes. Refractive Corneal Surg 1993; 9: S76S81.

9 Cursino JW, Fine BS. A histologic study of calcific and noncalcific band keratopathies. Am J Ophthalmol 1976; 82: 395-404.

10 O'Brart DPS, Gartry DS, Lohmann CP et al. Treatment of band keratopathy by excimer laser phototherapeutic keratectomy: surgical techniques and long term follow up. Br J Ophthalmol 1993; 77: 702-708.

11 Dighiero P, Boudraa R, Ellies P et al. Therapeutic photokeratectomy for the treatment of band keratopathy. J Fr Ophtalmol 2000; 27: 345-349.

12 Amano S, Oshika T, Tazawa Y et al. Long-term follow-up of excimer laser phototherapeutic keratectomy. Jpn J Ophthalmol 1999; 43: 513-516.

13 Amm M, Duncker GI. Refractive changes after phototherapeutic keratectomy. J Cataract Refractive Surg 1997; 23: 839-844.

14 Dogru M, Katakami C, Miyashita M et al. Ocular surface changes after excimer laser phototherapeutic keratectomy. Ophthalmology 2000; 107: 1144-1152.

15 Salah T, El Maghraby A, Waring III GO. Excimer laser phototherapeutic keratectomy before cataract extraction and intraocular lens implantation. Am J Ophthalmol 1996; 122: $340-348$. 